\title{
Tympanic Membrane Perforation and COVID-19
}

Firouzabadi FD ${ }^{1,2,3 *}$, Firouzabadi $\mathrm{MD}^{1,2 *}$, Moosaie $\mathrm{F}^{1,2,3 \#}$, Yoosefi $\mathrm{N}^{4 \#}$, Ramezanpour $\mathrm{S}^{5}$, Babaniamansour $\mathbf{S}^{6}$, Rafiee $\mathbf{S}^{1,2}$, Roomiani $\mathbf{M}^{1,2}$ and $\mathrm{J}$ ahandideh $\mathrm{H}^{1,2}$

${ }^{1}$ ENT and Head \& Neck Research Center, The Five Senses Institute, Iran University of Medical Sciences, Tehran, Iran

${ }^{2}$ Department of Otolaryngology-Head and Neck Surgery, Firoozgar Hospital, Iran University of Medical Sciences, Tehran, Iran

${ }^{3}$ Endocrinology and Metabolism Research Center (EMRC), Vali-Asr Hospital, School of Medicine, Tehran University of Medical Sciences, Tehran, Iran

${ }^{4}$ Department of Cellular and Physiological Sciences, Faculty of medicine, University of British Columbia, Canada

${ }^{5}$ Department of Radiology and Biomedical Imaging, University of California, San Francisco, USA

${ }^{6}$ School of Medicine, Islamic Azad University Tehran

Faculty of Medicine, Tehran, Iran

"Contributed Equally to this Work

*Corresponding author: Hesam J ahandideh, ENT and Head \& Neck Research Center, The Five Senses Institute, Iran University of Medical Sciences, Tehran, Iran

Maryam Roomiani, ENT and Head \& Neck Research Center, The Five Senses Institute, Iran University of Medical Sciences, Tehran, Iran

Received: April 13, 2021; Accepted: May 05, 2021;

Published: May 12, 2021

\section{Introduction}

The Coronavirus Disease 2019 (COVID-19) pandemic, caused by the Severe Acute Respiratory Syndrome Coronavirus 2 (SARSCoV-2) infection, first emerged from Wuhan, China in late 2019 and demonstrates several pre-established and new symptoms and manifestations [1-3]. An emerging area, in which there is little information available, is the COVID-19-related otologic manifestations. Though previous studies have demonstrated otalgia and vertigo as the first presentation in COVID-19 patients, there is still no clear image of the spectrum of otologic symptoms of COVID-19 [4,5]. Furthermore, to the best of our knowledge, no paper has reported Tympanic Membrane Perforation (TMP) after COVID-19 patients that can be subsequent to bacterial superinfection.

\section{Case Presentation}

A 61-year-old female without any past medical history or any trauma to ear during childhood or adultness or any drug use presented in the emergency department of Shahid Modares, Saveh, Iran. With the chief complaints of otalgia and a sensation of fullness in left ear, that she claimed had started one week after remission from COVID-19 symptoms. Three weeks before this otologic problem, she was admitted to the hospital due to fever, headache, dyspnea, cough and gastrointestinal symptoms, which persisted for 14 days. She also reported Otorrhea at the first admission that did not receive any eardrop. She was then admitted to the hospital and received Hydroxychloroquine 200mg PO q12h and Oseltamivir $75 \mathrm{mg}$ PO q12h, both for the duration of five days for treatment of COVID-19, after a confirmed COVID-19 diagnosis through Reverse Transcription-Polymerase Chain Reaction (RT-PCR) assay. Then, she was discharged without any signs and symptoms except for weakness. Chest Computed tomography (CT) scan had the pattern normally observed in COVID-19 patients. Her vital signs were also reported to be normal. Upon second admission, she was afebrile, not pale, anicteric, acyanotic and without any past medical history of olfactory and gustatory dysfunction. There were no palpable lymphnodes, paedal edema or organomegaly witnessed. Chest was clear with bilateral air entry equal, normal heart sounds and no added sounds. Upon further examination, both pinnae appeared normal. Otoscopy of the ear revealed clear external auditory canal and central perforation in the pars tensa with regular margins occupying the posteroinferior quadrant and normal level of middle ear mucosa. Weber test lateralized to the affected side and Rinnie was negative on that side. The patient was examined by the auditory specialist and found to have a $50 \%$ posteroinferior quadrant left-sided TMP. The consultant obtained a temporal bone CT scan and audiogram. CT scan (axial view) with thin cuts of the temporal bone revealed well-pneumatized mastoid regions without any density or secretion, normal middle ear without any density or secretion, and normal soft tissue density (Figure 2). The audiogram showed tympanic membrane
Austin J Radiol - Volume 8 Issue 4 - 2021

ISSN : 2473-0637 | www.austinpublishing group.com

Firouzabadi et al. (C) All rights are reserved
Citation: Firouzabadi FD, Firouzabadi MD, Moosaie F, Yoosefi N, Ramezanpour S, Babaniamansour S, et al. Tympanic Membrane Perforation and COVID-19. Austin J Radiol. 2021; 8(4): 1134 


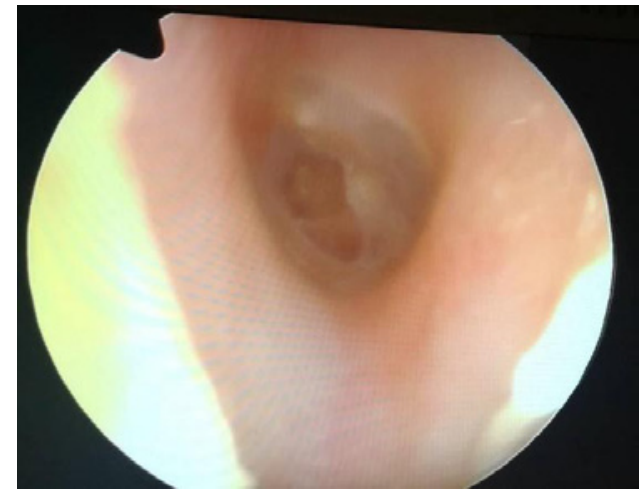

Figure 1: A perforated tympanic membrane over central part of right eardrum was noted by otoscope examination.
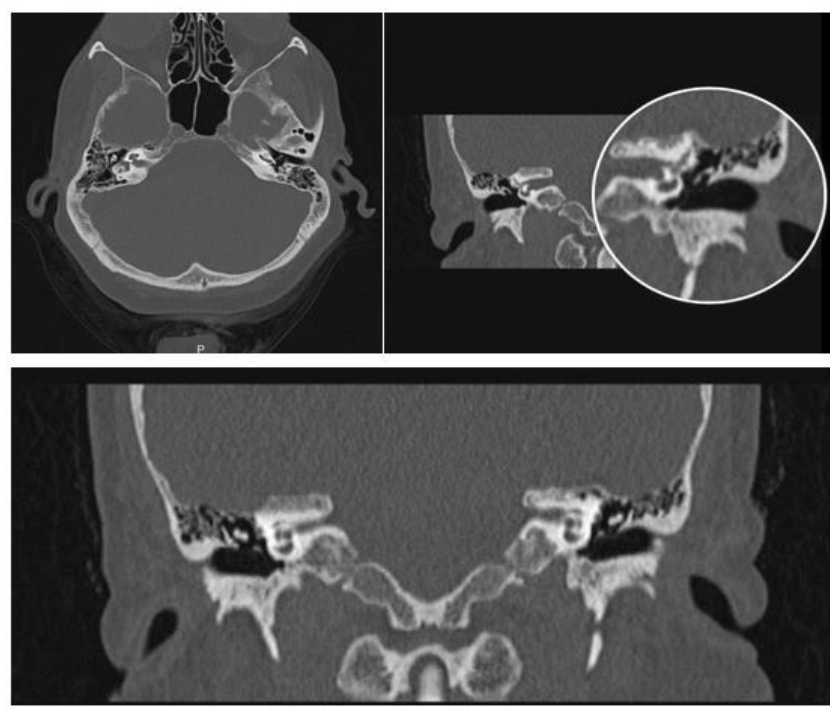

Figure 2: Computed tomography (axial view) with thin cuts of the temporal bone revealed well-pneumatized mastoid regions without any density or secretion and normal middle ears without any density or secretion.

velocity was found to be decreased in the high and low frequencies. The patient was elected for tympanoplasty without mastoidectomy 2 months as TMP was not fully healed (Figure 1). During her first day of visit, she was retested with PCR assay for COVID-19, and the result was found to be negative during the second hospitalization.

\section{Outcomes and Follow-up}

Tympanoplasty was performed without mastoidectomy by postauricular approach that was repaired via fascia tempolaris graft. Ossicular chains were checked intraoperatively and were found to be normal. She was advised to keep the affected ear dry and analgesics were given for pain relief. The patient's postoperative course was unremarkable, the TMP was healed and an audiogram performed 2 months later was normalized.

\section{Discussion}

While the incidence of TMP is increasing in developing countries [6] due to malnutrition, overcrowding, and frequent upper respiratory tract infections $[6,7]$, it is encouraged by poverty and the ignorance of the health governance to this health defect. Accordingly, clinical features of TMP such as severe tinnitus, vertigo, sensorineural hearing loss, hemorrhagic otorrhea, and facial paralyses need special attention and there should be appropriate infrastructure established for patients to be referred to an otorhinolaryngologist by the general practitioners and family physicians [8-10].

Recent studies have suggested SARS-CoV-2 virus being found in the middle ear canal and mastoid process on biopsy [11]. Hearing loss is also reported in COVID-19 patients raising the possibility of developing neuropathy as a side effect of COVID-19 infection, similar to other viral diseases such as measles, mumps and meningitis. While, the presence of the virus in these areas are observed, literature search revealed no reported cases of TM perforation related to COVID-19 patients. Importantly, the pathologies of the external and middle ear with tympanic perforation are rarely of viral origin and typically from herpes zoster [12]. This perforation is subsequent to bacterial superinfection in most of the time. Furthermore, it is possible that our patient's tympanic membrane perforation was unrelated to the Covid-19; however, this is unlikely considering the timing of the perforation despite the absence of any upper respiratory tract symptoms or other likely cause of the otitis.

The present case report is among the first to report any associations between COVID-19 and TMP. We report here an unusual cause of acute TMP, which followed a remission of COVID-19 infection, while highlighting the need to add a separated category for TMP cases, named COVID-19-associated TMP.

While common in otolaryngological practice, TMPs can have infective causes, traumatic causes or be a consequent of chronic eustachian tube disorders $[10,13]$. Although she reported she had otorreha during the course of COVID-19 infection, it could be subsequent to bacterial superinfection. Also, our patient however did not report any history of slap injuries, scuba diving experiences, or entry of foreign bodies.

Otalgia, malodorous otorrhea, and hearing loss are the most frequent presenting symptoms regarding cholesteatomas that is a benign collection of keratinized squamous epithelium within the middle ear [14]. Our patient's otoscopy did not show any evidence to support cholesteatomas even though she had otalgia and a sensation of fullness in left ear. Moreover, she did not have ossicular chain disruption, oval window rupture or evidence of facial nerve injury to support traumatic TMP $[15,16]$.

Although previous studies showed that Hydroxycholorochin had the potential of causing vestibulocochlear toxicity [17], our patient did not demonstrate any signs or symptoms of vestibulocochlear toxicity; the result of audiogram showed normal vestibulocochlear system.

Our patient, despite having no medical history and normal laboratory results after the second hospitalization, showed TMP presentation.

Overall, hearing loss due to otology involvement can be subsequent to bacterial superinfection after remission of COVID-19, which is difficult to detect in early stages and requires swift attention. Therefore, further global clinical studies with longer follow-ups are needed to determine if SARS-CoV-2 can cause TMP and any long- 
term ear diseases.

\section{References}

1. Goudarzi S DFF, Mahmoudzadeh F, Aminimoghaddam S. Pulmonary embolism in a pregnant woman with COVID-19 infection: a case report. clinical case reports. 2021.

2. Goudarzi S, Dehghani Firouzabadi F, Dehghani Firouzabadi M, Rezaei N. Cutaneous lesions and COVID-19: Cystic painful lesion in a case with positive SARS-CoV-2. Dermatologic Therapy. 2020; 33: e14266.

3. Saberian $P$, Mireskandari SM, Baratloo A, Hasani-Sharamin $P$ Babaniamansour S, Aliniagerdroudbari E, et al. Antibody Rapid Test Results in Emergency Medical Services Personnel during COVID-19 Pandemic; a Cross Sectional study. Arch Acad Emerg Med. 2021; 9: e2.

4. Dehghani Firouzabadi M, Dehghani Firouzabadi F, Goudarzi S, Jahandideh $\mathrm{H}$, Roomiani M. Has the chief complaint of patients with COVID-19 disease changed over time? Medical Hypotheses. 2020; 144: 109974.

5. Firouzabadi FD, Firouzabadi MD, Ghalehbaghi B, Jahandideh H, Roomian M, Goudarzi S. Have the symptoms of patients with COVID-19 changed over time during hospitalization? Med Hypotheses. 2020; 143: 110067.

6. Ibekwe TS IG, Nwaorgu OG. Tympanic membrane perforation among adults in West Africa. Otology \& neurotology : official publication of the American Otological Society, American Neurotology Society and European Academy of Otology and Neurotology. 2007; 28: 348-352.

7. Ologe FE, Nwawolo CC. Prevalence of chronic suppurative otitis media (CSOM) among schoolchildren in a rural community in Nigeria. The Nigerian postgraduate medical journal. 2002; 9: 63-66.

8. Cahali S, da Silva FB, Machado MC, da Silva DA, Reforeme OMR, Cahali
MB. Middle ear squamous papilloma: report of a case and literature review. Brazilian journal of otorhinolaryngology. 2005; 71: 396-398.

9. ML H. Middle ear, tympanic membrane, perforations. 2006.

10. O'Handley JG TE, Shah AR. Otorhinolaryngology, Textbook of family medicine. Philadelphia: Saunders. 2011.

11. Frazier KM, Hooper JE, Mostafa HH, Stewart CM. SARS-CoV-2 Virus Isolated From the Mastoid and Middle Ear: Implications for COVID-19 Precautions during Ear Surgery. JAMA Otolaryngology Head \& Neck Surgery. 2020; 146 : 964-966.

12. Heidi M Crane, Greg Davis, Karen Mark, Harrington RD. Tympanic Membrane Perforation: An Unusual Complication of Varicella-Zoster Virus Infection. 2008

13. B I. Hearing loss. Med Clin North Am. 2010; 94: 973-988.

14. Hsing-Won Wang, P-ZC, Fei-Peng Lee. A Tympanic Membrane Cholesteatoma: A Case Report and Literature Review Science Repository. 2019.

15. Mirza S, Richardson H. Otic barotrauma from air travel. The Journal of laryngology and otology. 2005; 119: 366-370.

16. Lou ZC, Lou ZH, Zhang QP. Traumatic tympanic membrane perforations: a study of etiology and factors affecting outcome. American journal of otolaryngology. 2012; 33: 549-555.

17. Fernandes MRdN, Soares DBR, Thien Cl, Carneiro S. Hydroxychloroquine ototoxicity in a patient with systemic lupus erythematosus. An Bras Dermatol. 2018; 93: 469-470. 